Proc. Indian Acad. Sci., Vol. 87 A (E \& P Sciences), No. 3, March 1978, pp. 1-12, (C) printed in India.

\title{
Nonlinear Rayleigh-Taylor instability in partially ionized plasma and the equatorial spread- $F$
}

\author{
R K JAIN and A C DAS \\ Physical Research Laboratory, Ahmedabad 380009 \\ MS received 25 June 1977; revised 28 December 1977
}

\begin{abstract}
The nonlinear evolution of the collisional gravitation induced RayleighTaylor (R-T) instability in the equatorial $F$ region is investigated taking into account the finite larmor radius (FLR) effects and the complete ion inertial term in ion equation of motion. A special class of coherent weakly nonlinear modes as solutions to the wave equation describing R-T instability driven modes is obtained. The leading nonlinear effects in the wave equation are found to appear through $V_{L}$, the ion diamagnetic drift which essentially gives the FLR corrections. It is shown that the R-T modes in the equatorial $F$ region can evolve into coherent, nonlinear, almost sinusoidal, stationary wave structures. These structures are found to travel with a constant phase velocity and to have slightly distorted sinusoidal shapes. These results seem to have a good agreement with many of the recent rocket and satellite observations of the equatorial spread $F$ irregularities.
\end{abstract}

Keywords. Rayleigh-Taylor instability; diamagnetic drift; phase coherence; FLR corrections; irregularities.

\section{Introduction}

The well known phenomenon of equatorial spread $F$ which is attributed to the fieldaligned electron density irregularities occurring in the equatorial $F$ region, has been the subject of both theoretical and experimental investigations by many authors in recent past (Booker and Wells 1938; Dungey 1956; Herman 1966; Reid 1968; Farley et al 1970; Balsley et al 1972; Hudson et al 1973; Farley 1974; Hudson and Kennel $1974 \mathrm{a}, \mathrm{b}, 1975 \mathrm{a}, \mathrm{c})$ and yet the processes responsible for producing these irregularities are not completely understood.

The equatorial spread $F$ is essentially a night time phenomenon associated with a post sunset rise of the $F$ layer. A most important and obvious source of free energy for driving an instability on the bottomside of the night time $F$ layer is the sharp vertical plasma density gradient. The density gradient-driven plasma instability or cross-field instability which is dominated in collisional plasma (Reid 1968; Cunnold 1969), does not appear to be appropriate for $F$ region because of negligible effects of collisions at such height (Farley et al 1970). It was, however, suggested by Reid (1968) that the electric field generated by cross-field instability in the $E$ region could be conducted up along equipotential magnetic field lines to the $F$ region. Farley et al (1970) have shown, however, that only large scale irregularities $(>1 \mathrm{~km})$ could be transported from $E$ to $F$ region. Furthermore, while such a theory may have significance at high lattitudes, it is not clear that it is relevant to equatorial region. 
After sunset, the $F$ region plasma resembles a slab of inhomogeneous plasma which is known to be unstable due to Rayleigh-Taylor (R-T) instability. Dungey (1956) was the first to suggest that the source of equatorial $F$-region irregularities could be a gravitational instability of the underside of the $F$ layer. Many properties of long wavelength irregularities $\left(\lambda_{\perp}>\rho_{l}\right.$, ion Larmor radius) below the $F$ region maximum can actually be explained in terms of this gravitation induced Rayleigh-Taylor instability (Johnson and Hulburt 1950; Dungey 1956; Balsley et al 1972; Hudson et al 1973; Hudson and Kennel 1975 a,c)

Chaturvedi and Kaw (1975) have obtained nonlinear steady state solutions of equations describing $\mathbf{R}-\mathrm{T}$ mode instability. It is shown that $\mathrm{R}-\mathrm{T}$ instability driven mode can evolve into a coherent, travelling stationary wave having a constant real phase velocity $u$, through nonlinear interactions with the shorter wavelength $F L R$ stable oscillatory modes. For $u<V_{L}$ where $V_{L}$ is the ion diamagnetic drift, they have also shown that the R-T modes evolve into almost sinusoidal solutions. Their results could explain some of the observations of the equatorial $F$ region irregularities (Dyson $e t$ al 1974; Kelley et al 1976).

In all the linear and nonlinear theories of spread $F$ irregularities mentioned above, the linear convection part $\left(V_{0} \cdot \nabla\right) \tilde{V}$ of ion inertial term has been neglected. However, the nonlinear theory modifies the phase-velocity of the wave because of the generation of higher harmonics and then the phase velocity may be comparable with or even smaller than $V_{L}$. In this case it becomes necessary to include the convective $\operatorname{part}\left(\mathbf{V}_{\mathbf{0}} \cdot \mathbf{\nabla}\right) \tilde{\mathbf{V}}$ in the ion equation of motion. Chaturvedi and Kaw (1975) had to assume $u<V_{L}$ in their nonlinear theory in order to explain some features of spread $F$ observations even though they had neglected the convective term. It is felt that the convective motion with thermal correction may play an important role for the stabilization of the long-wave-length R-T modes in the $F$ region.

In this paper a nonlinear theory of the collisional $R-T$ instability is presented accounting for FLR (Rosenbluth et al 1962) and ion viscous effects, which differs from the earlier theories in the sense that it includes in the ion equation of motion the convective part also. Following a method which is different from one that is used by Chaturvedi and Kaw (1975) a special class of coherent, weakly nonlinear R-T modes in the equatorial spread $F$ region is discussed. The basic set of equations, assumptions and a brief description of the equilibrium configuration are given in the next section. The linear theory is discussed in section 3 and the derivation of an ordinary nonlinear differential equation from the set of nonlinear partial differential equations describing R-T instablility driven modes is considered in section 4 . Nonlinear solution to this wave equation, the corresponding dispersion relation and the physical conditions under which the solutions are valid, are discussed in section 5 . Finally, the discussions and concluding remarks of the theoretical results with their relevance to the experimental observations are presented in last section.

\section{Basic equations}

For the wavelength of the perturbations greater than either ion gyroradius or ion mean-free path, the macroscopic fluid equations are adequate to describe the $F$ region plasma. We choose the following rectangular coordinate system appropriate to the equatorial $F$ region: $z$-axis points along the direction of earth's magnetic field; $y$-axis 
is taken along the west-east direction and $x$-axis points vertically upward. The background density gradient is thus along the $x$-axis and antiparallel to the acceleration due to gravity g. In the frame of two-fluid theory, the equatorial $F$ region plasma, is, therefore, governed by the continuity and momentum transfer equations for electrons and ions which are given by

$$
\begin{aligned}
& \frac{\partial n}{\partial t}+\nabla \cdot(n \mathbf{v})=0 \\
& 0=-\frac{e}{m}\left(\mathbf{E}+\frac{\mathbf{v} \times \mathbf{B}}{c}\right)-\frac{K T_{e}}{m} \frac{\nabla n}{n} \\
& \frac{\partial N}{\partial t}+\nabla \cdot(N \mathbf{V})=0 \\
& \frac{\partial \mathbf{V}}{\partial t}+(\mathbf{V} \cdot \nabla) \mathbf{V}=\frac{e}{M}\left(\mathbf{E}+\frac{\mathbf{V} \times \mathbf{B}}{c}\right)-v_{\iota} \mathbf{V}+\mathbf{g} \\
& \quad-\frac{K T_{i}}{M} \frac{\nabla N}{N}+\mu \nabla^{2} \mathbf{V}
\end{aligned}
$$

where $n$ and $N$ are the number densities of electrons and ions respectively; $E=-\nabla p$ is the electric field and $\phi$ is the electrostatic potential; $\mathbf{v}$ and $\mathbf{V}$ represent the electron and ion velocities; $m$ and $M$ refer to the electron and ion masses respectively; $\mu$ is the coefficient of ion viscosity and $v_{t}$ is the ion-neutral collision frequency. All other symbols have their conventional meanings.

\subsection{Basic assumptions}

In writing down the above equations and in subsequent discussions, the following assumptions and approximations are made: (i) The electron collision frequency $\nu_{\text {e }}$ is neglected because $\nu_{e} \& \Omega_{e}$, the electron gyrofrequency $\left(\Omega_{e}=e B / m c\right)$; (ii) The electron inertia has been ignored since $\Omega_{e}$ is much larger than the Doppler-shifted wave frequency; (iii) The wave electric field is essentially electrostatic, i.e., $E=-\nabla \phi$ (in agreement with observations, Holtet et al 1975), because the plasma pressure is much smaller than the ambient magnetic energy density and $c k / \omega_{p e}>1$ for wavelength of interest ( $\omega_{p e}$ is electron plasma frequency and $k$ is wave number); (iv)The electrons and ions are assumed to be isothermal; (v) The ion drag which is unim portant at night (Rishbeth and Garriott 1969), is neglected; (vi) The recombination is assumed to be negligible which is valid for the processes that occur faster than the several hour time scale of the nighttime $F$ region; (vii) The waves are assumed to propagate exactly perpendicular to the magnetic field, since these waves experience least damping due to diffusion; (viii) The neutrals are considered completely at rest.

Since we are interested in long wavelength $\mathrm{R}-\mathrm{T}$ modes, we invoke the quasineutrality condition namely

$$
n \approx N .
$$




\subsection{Equilibrium configuration}

In presence of collisional transport effects, both the sinks and sources are neglected. We will assume that there is only upward vertical drift of the particles due to zeroth order eastwest dc electric field $\mathbf{E}_{\mathbf{0}}$. This zero-order electric field is generated due to the steady motions of neutral winds in the earth's magnetic field at $E$ region heights. This is termed as 'dynamo field'. Since the lines of force of earth's magnetic field are equipotential, this electric field is transferred from the $E$ region heights at low latitude to $F$ region heights in the equatorial region. In zero-order stationary equilibrium state, eqs (1) to (4) yield the electron and ion zero order equilibrium drift velocities in horizontal and vertical directions as

$$
\begin{aligned}
& v_{o x}=v_{D} ; V_{o x}=v_{D}-\frac{\nu_{t}}{\Omega_{l}}\left(V_{L}+\frac{g}{\Omega_{l}}\right) \\
& v_{o y}=-v_{L} ; \quad V_{o y}=V_{0}=\frac{v_{t}}{\Omega_{l}} v_{D}+V_{L}+\frac{g}{\Omega_{l}}
\end{aligned}
$$

where $v_{D}=c\left[\left(\mathrm{E}_{0} \times \mathbf{B}\right) / B^{2}\right]$ is the drift velocity of the particles and $v_{L}=v_{\text {th }} \rho_{e} / L$ and $V_{L}=V_{\mathrm{th}} \rho_{t} / L$ are the electron and ion diamagnetic drifts respectively. $\rho_{e}=$ $v_{\text {th }} / \Omega_{\text {e }}$ and $\rho_{t}=V_{\mathrm{th}} / \Omega_{l}$ are the electron and ion larmor radii respectively; $v_{\mathrm{th}}=$ $\left(K T_{e} / m\right)^{1 / 2}$ and $V_{\mathrm{th}}=\left(K T_{\mathrm{t}} / M\right)^{1 / 2}$ are respectively the electron and ion thermal velocities and $L$ is the background density scale length defined by

$$
L^{-1}=\frac{1}{n_{0}} \frac{d n_{0}}{d x}
$$

The finite larmor radius effects are introduced through $v_{L}$ and $V_{L}$. The ion viscous diffusion flux along the density gradient, which is proportional to $\left(v_{t} / \Omega_{l}\right) \rho_{l} / L$ is also negligible and hence has not been considered. $\nu_{l} / \Omega_{l}<10^{-3}$ and $V_{L}$ is typically $\gtrsim 0.2 \mathrm{~m} / \mathrm{s}$ above $250 \mathrm{~km}$.

\section{Linear theory}

Let us define each variable $q$ consisting of two parts

$$
q=q_{0}+\tilde{q}
$$

where $q_{0}$ denotes the equilibrium value and $\tilde{q}$ is the perturbation which we assume to be horizontally propagating low frequency wave of the form exp $[i(k y-\omega t)]$. Linearizing eqs (1) to (4) and then eliminating all except density variables and using condition (5), we obtain the linear dispersion relation as

$$
\omega^{2}-\omega\left(k V_{0}+k V_{L}-i v_{l}\right)+\left\{k^{2} V_{L} V_{0}-i k V_{L^{\nu_{l}}}+\left(v_{l} v_{D}+g\right) / L\right\}=0
$$

where $\omega$ and $k$ are the frequency and wave number of the wave respectively. Vis- 
cous effects have been neglected because the term containing $\mu$ is much smaller than others in magnitude as discussed before.

For $\nu_{i}=0$, the dispersion relation becomes

$$
\omega^{2}-\omega\left(k V_{0}+k V_{L}\right)+\left(k^{2} V_{L} V_{0}+g / L\right)=0
$$

The phase velocity $u$ and the growth rate $\gamma$ are then given by

$$
u=\frac{1}{2}\left(V_{0}+V_{L}\right)
$$

and

$$
\gamma=\left[(g / L)\left(1-k^{2} L g / \Omega_{i}^{2}\right)\right]^{1 / 2}
$$

If the effects of the ion diamagnetic drift and the ion gravitational drifts are neglected for large wavelength waves, then the dispersion relation (11) reduces to $\omega^{2}=-g / L$ which is the usual dispersion relation for Rayleigh-Taylor mode in collisionless plasma. In the collisional limit $\left(\nu_{i} \neq 0\right)$, the solutions of the dispersion eq. (10) are given by

$$
\begin{aligned}
\omega== & \frac{1}{2}\left(k V_{0}+k V_{L}-i \nu_{l}\right) \pm \frac{1}{2}\left[\left(k V_{0}+k V_{L}-i v_{l}\right)^{2}\right. \\
& \left.-4\left\{k^{2} V_{L} V_{0}-i k V_{L^{\nu}}+\left(\nu_{l} v_{D}+g\right) / L\right\}\right]^{1 / 2} .
\end{aligned}
$$

For spread $F$ region $L \approx 40 \mathrm{~km}$ (Hanson and Sanatani 1973, Kelley and Carison 1974) and $\nu_{l} \approx 10^{-1}$ to $10^{-2} \mathrm{~s}^{-1} . \quad V_{\text {th }}=1 \mathrm{~km}$ and therefore

$$
\nu_{l}>g / L \text { or } k V_{L} \text { or } k V_{0}
$$

for modes with wavelengths much larger than the larmor radius $\rho_{l}(\approx 10$ meters $)$.

Then it is seen from (12) that one root gives a completely damped mode because of collisions while the other root provides an unstable mode. The phase velocity and the growth are given by

$$
U=\omega_{r} / k=V_{L}+\left(\nu_{l} v_{D}+g\right)^{2} / \Omega_{i} L \nu_{l}^{2}
$$

and

$$
\gamma=\left(\nu_{i} \nu_{D}+g\right) / L \nu_{i}
$$

where $\omega_{r}$ and $\gamma$ are the real and imaginary parts of $\omega$. The growth rate $\gamma$ is in agreement with the collisional Rayleigh-Taylor mode growth rate obtained by Balsley et al (1972) and Hudson and Kennel $(1975 \mathrm{a}, \mathrm{c})$. Only difference, as expected, is that the growth has a contribution from the vertical drift of the particles and it becomes quite large if the velocity due to the electric field is enhanced considerably. It also, becomes obvious from (14) and (15) that the growth rate as well as the phase velocity of the collisional R-T instability driven modes increase with height because $v_{i}$ decreases with altitude. 
In the situation when the electric field is absent, it is obvious that the growth rate is purely due to unstable long wavelength collisional R-T mode and it increases with increasing height. It, therefore, suggests that spread $F$ must occur at higher height. The inclusion of the electric field has changed this character. If the electric field is enhanced (a situation which can occur in the region of interest), then the growth rate term involving the electric field can become considerable and therefore, the spread $F$ can occur at a lower height. The relevance of these results with the observations will be discussed in a later section.

\section{Wave equation for nonlinear $\mathrm{R}-\mathrm{T}$ modes}

Substituting (9) into (1) to (4) and using the zero-order equilibrium state $q_{0}$ and the condition (5), we get the following set of equations for perturbed quantities $\tilde{q}$.

$$
\begin{aligned}
& 0=\frac{c}{B} \nabla \phi-\frac{\tilde{\mathbf{v}} \times \mathbf{B}}{B}-\frac{v_{\mathrm{th}}^{2}}{\Omega_{e}}\left[\frac{\nabla \tilde{n}}{n}-\frac{\tilde{n}}{n L} \hat{\mathbf{x}}\right] \\
& \frac{\partial \tilde{n}}{\partial t}+\frac{\partial}{\partial x}\left(n \tilde{v}_{x}\right)+\frac{\partial}{\partial y}\left(n \tilde{v}_{y}\right)+v_{o y} \frac{\partial \tilde{n}}{\partial y}=0 \\
& \frac{1}{\Omega_{l}}\left(\frac{\partial}{\partial t}+V_{o} \frac{\partial}{\partial y}\right) \tilde{\mathbf{v}}=-\frac{c}{B} \nabla \phi+\frac{\tilde{\mathbf{v}} \times \mathbf{B}}{B}-\frac{\nu_{l}}{\Omega_{l}} \tilde{\mathbf{v}} \\
& -\frac{V_{\mathrm{th}}^{2}}{\Omega_{l}}\left(\frac{\nabla \tilde{n}}{n}-\frac{\tilde{n}}{n L} \hat{\mathbf{x}}\right)+\left(\mu / \Omega_{l}\right) \nabla^{2} \tilde{\mathbf{V}} \\
& \frac{\partial \tilde{n}}{\partial t}+\frac{\partial}{\partial x}\left(n \tilde{V}_{x}\right)+\frac{\partial}{\partial y}\left(n \tilde{V}_{y}\right)+V_{o y} \frac{\partial \tilde{n}}{\partial y}=0
\end{aligned}
$$

where $\phi$ is the electrostatic potential perturbation and $\hat{x}$ is the unit vector along the $\mathbf{x}$-direction, The velocities $\mathbf{v}$ and $\mathbf{V}$ have components only in perpendicular direction to $\mathbf{B}$ (as $k_{11}=0$ in accordance with the assumption vii). Combining electron continuity eq. (17) and equation of motion (16), we get

$$
\frac{\partial \tilde{n}}{\partial t}-\frac{n_{0}}{L} \frac{c}{B} \frac{\partial \phi}{\partial y}
$$

which shows that the density changes locally because of $\tilde{\mathbf{E}} \times \mathbf{B}$ motion of electrons along the direction of density gradient $\left(\nabla n_{0}=n_{0} / L\right)$. The solution of ion equation of motion (18) for the components of ion velocity $\tilde{V}_{x}$ and $\tilde{V}_{y}$ and then substitution of these into ion equation for continuity (19), yield the equation for ion density change due to motion of ions as 


$$
\begin{aligned}
& \frac{\partial \tilde{n}}{\partial t}+\frac{n_{0} V_{L}}{\Omega_{i} L} \frac{\partial}{\partial t}\left(\log \frac{n}{n_{0}}-\frac{\tilde{n}}{n}\right)-\frac{n_{0}}{L} \frac{c}{B} \frac{\partial \phi}{\partial y}+\frac{g^{\prime}}{\Omega_{i}} \frac{\partial \tilde{n}}{\partial y} \\
& \quad-\frac{1}{\Omega_{l}}\left[\left(\frac{\partial}{\partial t}+V_{0} \frac{\partial}{\partial y}-\mu \frac{\partial^{2}}{\partial y^{2}}\right) \frac{\partial}{\partial y}\left(L V_{L} \frac{\partial \tilde{n}}{\partial y}-\frac{c}{B} n \frac{\partial \phi}{\partial y}\right)\right. \\
& +\nu_{l} \frac{\partial}{\partial y}\left(L V_{L} \frac{\partial \tilde{n}}{\partial y}+\frac{c}{B} n \frac{\partial \phi}{\partial y}\right) \\
& \left.\quad-\frac{\partial}{\partial y}\left\{\left(\frac{c}{B} \frac{\partial \phi}{\partial y}+\frac{L V_{L}}{n} \frac{\partial \tilde{n}}{\partial y}\right)\left(\frac{\partial}{\partial t}+V_{0} \frac{\partial}{\partial y}-\mu \frac{\partial^{2}}{\partial y^{2}}\right) \tilde{n}\right\}\right]=0
\end{aligned}
$$

where $g^{\prime}=g+v_{i} v_{D}$. In simplifying this equation the terms which contain the square and product of $\omega\left|\Omega_{i}, k\right| \Omega_{t}$ and $v_{i} \mid \Omega_{l}$ and other smaller terms have been neglected in comparison with other leading terms. In this equation the third term describes the generation of density fluctuation because of $\tilde{\mathbf{E}} \times \mathbf{B}$ effect in the direction of perpendicular density gradient similar to electrons. The fourth term represents the density fluctuation arising because of the gravitational drift of ions and the last term gives the contribution due to the effects of finite inertia, ion collisions and ion viscosity. Combining eq. (20) for electrons and eq. (21) for ions, we obtain the partial differential equation for the R-T modes as

$$
\begin{aligned}
& \left(\frac{\partial}{\partial t}+V_{0} \frac{\partial}{\partial y}-\mu \frac{\partial^{2}}{\partial y^{2}}\right)\left(n \frac{\partial^{2} \tilde{n}}{\partial y \partial t}+n_{0} V_{L} \frac{\partial^{2} \tilde{n}}{\partial y^{2}}+\frac{\partial \tilde{n}}{\partial t} \frac{\partial \tilde{n}}{\partial y}\right) \\
& +v_{t} \frac{\partial}{\partial y}\left(n \frac{\partial \tilde{n}}{\partial t}+n_{0} V_{L} \frac{\partial \tilde{n}}{\partial y}\right)-\frac{\partial}{\partial y}\left[\left(\frac{\partial \tilde{n}}{\partial t}\right)^{2}-\frac{n_{0} V_{L} V_{0}}{n}\left(\frac{\partial \tilde{n}}{\partial y}\right)^{2}\right. \\
& \left.+V_{0}\left(1+\frac{V_{L}}{V_{0}} \frac{n_{0}}{n}\right) \frac{\partial \tilde{n}}{\partial y} \frac{\partial \tilde{n}}{\partial t}-\mu\left(\frac{\partial \tilde{n}}{\partial t}+\frac{n_{0} V_{L}}{n} \frac{\partial \tilde{n}}{\partial y}\right) \frac{\partial^{2} \tilde{n}}{\partial y^{2}}\right] \\
& =n_{0} g^{\prime} \frac{\partial \tilde{n}}{\partial y}+\left(\frac{n_{0}}{L}\right)^{2} V_{L} \frac{\partial}{\partial t}\left(\log \frac{n}{n_{0}}-\frac{\tilde{n}}{n}\right) .
\end{aligned}
$$

The solution of the above equation will describe the nonlinear evolution of R-T modes. However, it is quite difficult to solve this nonlinear equation and therefore, we will not derive a simpler equation by making a suitable transformation such that all the oscillating quantities are functions of only $s-u t$, where $s$ is the coordinate along the direction of wave propagation. In case of periodic modes it is convenient to introduce the phase of oscillation as

$$
\xi=k y-\omega t
$$


This then reduces (22) into an ordinary differential equation in $\xi$, which after one integration and some algebra yields

$$
\begin{aligned}
& \left(u-V_{0}\right)\left\{u(1+\eta)-V_{L}\right\} \frac{d^{2} \eta}{d \xi^{2}}+\frac{\left(u-V_{0}\right) V_{L}}{1+\eta}\left(\frac{d \eta}{d \xi}\right)^{2} \\
& -\frac{\nu_{l}}{k}\left(u(1+\eta)-V_{L}\right) \frac{d \eta}{d \xi}-\frac{g^{\prime}}{k^{2} L} \eta \\
& +\mu k\left[\left\{u(1+\eta)-V_{L}\right\} \frac{d^{3} \eta}{d \xi^{3}}+\left(4 u-\frac{V_{L}}{1+\eta}\right) \frac{d \eta}{d \xi} \frac{d^{2} \eta}{d \xi^{2}}\right] \\
& +\frac{u V_{L}}{k^{2} L^{2}}\left(\log (1+\eta)-\frac{\eta}{1+\eta}\right)=0
\end{aligned}
$$

where $\eta=\tilde{n} / n_{0}$ and $u=\omega / k$. We will ignore $\mu$ for the ion viscosity term is found to be much smaller in magnitude compared to other terms below the equatorial $F$ region maximum. We further assume that $u \leqslant V_{0}$, consistent with our earlier discussions and that $\eta<1$ for weak nonlinearity. With the expansion of $\log (1+\eta)$ in the last term, the nonlinear eq. (24) of R-T modes becomes

$$
\begin{gathered}
\frac{d^{2} \eta}{d \xi^{2}}+\frac{V_{L}}{\left(u-V_{L}\right)} \frac{1}{\left(1+m_{0} \eta\right)(1+\eta)}\left(\frac{d \eta}{d \xi}\right)^{2}+\frac{\nu_{i}}{k\left(V_{0}-u\right)} \frac{d \eta}{d \xi} \\
+p^{2} \frac{\eta}{1+m_{0} \eta}-\frac{m p^{2}\left[1-(n / 3)+\left(\eta^{2} / 6\right)\right] \eta^{2}}{\left(1+m_{0} \eta\right)(1+\eta)}=0
\end{gathered}
$$

where

$$
\begin{aligned}
& m_{0}=u /\left(u-V_{L}\right) ; m=u V_{L} / 2 g^{\prime} L \\
& p^{2}=g^{\prime} /\left[k^{2} L\left(V_{0}-u\right)\left(u-V_{L}\right)\right] .
\end{aligned}
$$

Equation (25) is the exact nonlinear wave equation describing the low frequency and long wavelength R-T modes generated on the underside of the $F$ region maximum of the equatorial ionosphere. One can now readily see that the periodic solutions of this wave equation are possible only when the fourth term is a positive quantity i.e., $V_{L}<u<V_{0}$. This essentially requires that either $\nu_{l}>5 \times 10^{-2}$ for $L=40 \mathrm{kms}$ and $v_{D}=20 \mathrm{~m} / \mathrm{s}$ or $L>1 \mathrm{~km}$ for $\nu_{D}=20 \mathrm{~m} / \mathrm{s}$ and $\nu_{i}=10^{-1}$.

\section{Nonlinear solution of the wave equation}

For weakly nonlinear modes, $\eta \leqslant 1$ and the wave eq. (25) for weakly nonlinear R-T modes can, therefore, be written as 


$$
\begin{gathered}
\frac{d^{2} \eta}{d \xi^{2}}+\frac{V_{L}}{\left(u-V_{L}\right)} \frac{1}{\left(1+m_{0} \eta\right)(1+\eta)}\left(\frac{d \eta}{d \xi}\right)^{2}+\frac{\nu_{i}}{k\left(V_{0}-u\right)} \frac{d \eta}{d \xi} \\
+\frac{p^{2} \eta}{1+m_{0} \eta}-\frac{m p^{2}(1-\eta / 3)}{\left(1+m_{0} \eta\right)(1+\eta)} \eta^{2}=0 .
\end{gathered}
$$

In the weakly nonlinear regime, the amplitude and the phase of the wave vary slowly with $\xi$. We use the method of averaging of Bogoliubov and Mitropolsky (1961) to solve eq. (27). Let the solution be

$$
\eta=A \cos (p \xi+\Phi)
$$

where $A$ and $\Phi$ are slowly varying function of $\xi$. Following Bogoliubov and Mitropolsky (1961) we obtain the dependence of $A$ and $\Phi$ on $\xi$ as

$$
\begin{aligned}
& \frac{d A}{d \xi}=-\frac{v_{i} A}{2 k\left(V_{0}-u\right)} \frac{1+m_{0} A^{2} / 4}{1+m_{0} A^{2} / 2} \\
& \frac{d \Phi}{d \xi}=\frac{1}{2} \frac{A p}{1+m_{0} A^{2} / 2}\left\{1-\frac{8}{8} m_{0}\left(1-\frac{u V_{L}}{6 m_{0} g^{\prime} L}\right) A\right\} .
\end{aligned}
$$

In the nonlinear stationary regime, the amplitude gets saturated and does not vary with $\xi$, i.e., the saturated amplitude is given by

$$
\frac{d A}{d \xi}=\frac{\nu_{i} A}{2 k\left(V_{0}-u\right)}\left(1+\frac{u}{u-V_{L}} \frac{A^{2}}{4}\right)\left(1+\frac{u}{u-V_{L}} \frac{A^{2}}{2}\right)^{-1}=0
$$

The saturated amplitude $A_{0}$ can be obtained by neglecting the terms of order higher than $A^{3}$ in the expansion of $\left[1+\left(\frac{u}{u-V_{L}}\right) \cdot A^{2} / 2\right]^{-1}$. With this stationary amplitude, eq. (27) is satisfied by the oscillatory solution of the type

$$
\eta=A_{0} \cos (k y-w t+\Phi)
$$

if

$$
p^{2}=1
$$

i.e., $\quad\left(V_{0}-u\right)\left(u-V_{L}\right)=g^{\prime} / k^{2} L$

and if $\boldsymbol{u}=\boldsymbol{u}_{0}$ at stationary amplitude, then

$$
1-\frac{u_{0}}{u_{0}-V_{L}} \frac{A_{0}^{2}}{4}=0
$$

i.e. $\quad u_{0}=V_{L}\left(1+A_{0}^{2} / 4\right)$. 
It is to be noted that eq. (30) shows how the phase of the waves $\Phi$ changes slowly with $\xi$ in nonlinear state, while the amplitude of the waves becomes stationary and the change in phase can be obtained from the following equation

$$
\frac{d \Phi_{0}}{d \xi}=\frac{p}{2}\left[1-\frac{3}{8} \frac{u_{0}}{u_{0}-V_{L}}\left(1-\frac{\left(u_{0}-V_{L}\right) V_{L}}{6 g^{\prime} L}\right) A_{0}-\frac{u_{0}}{u_{0}-V_{L}} \frac{A_{0}^{2}}{2}\right] A_{0}
$$

where $\Phi_{0}$ corresponds to the value of $\Phi$ in nonlinear regime when the amplitude attains a constant value $A_{0}$. This corresponds to a situation in which coherent, small amplitude, stationary, almost sinusoidal oscillations satisfying both (33) and (34) exist in the system which supports R-T modes.

It is seen from eq. (35) that the phase of the waves changes slightly with $\xi$ which indicates that the modes develop finally into slightly distorted sinusoidal shapes rather than pure sinusoidal forms. Such slightly distorted wavelike structures have been observed and this result may, therefore, explain some observations of nonsinusoidal steepened wavelike structures of the ionization density irregularities in the equatorial spread $F$ (Dyson et al 1974; Kelley et al 1976). Equation (34) shows that the phase velocity of the waves in the nonlinear regime depends upon the nonlinear amplitude $A_{0}$ and also becomes greater than the ion diamagnetic drift velocity $V_{L}$ by a small factor $V_{L} A_{0}^{2} / 4$ as the amplitude of the mode increases as a result of the linear growth. This implies that the R-T modes will become stationary only when $\left(u-V_{L}\right) \approx V_{L} A_{0}^{2} / 4$, i.e., $u-V_{L}>0$, which is also the condition for periodicity.

The magnitude of different nonlinear terms appearing in wave eq. (27) can be estimated for the parameters appropriate to the region of interest. It is found that the leading term is one which is proportional to $(d n / d \xi)^{2}$ and depends upon the ion diamagnetic drift $V_{L}$. This clearly shows that the FLR corrections seem to be responsible for producing important nonlinearities which, in turn, lead to the stabilization of R-T modes through the nonlinear interaction of the waves.

Physically, the mechanism of the stabilization of growing R-T modes which evolve into coherent, almost sinusoidal travelling stationary waves in nonlinear regime, may be understood in the following manner. The fundamental unstable long wavelength R-T modes are first generated and then grow in amplitude. After a few growth period the amplitude becomes sufficiently large and produces higher harmonics which can get coupled with the fundamental through nonlinear interactions. The FLR effects reduce the growth rate and the nonlinear interaction gives rise to the contribution to the real frequency of the fundamental as well as the higher harmonics such that the phase velocity increases. When the phase velocities of these modes become greater than the ion diamagnetic drift $V_{L}$ in the nonlinear regime, the nonlinear interactions lead to the dissipation of energy associated with the fundamental to its higher harmonics. The higher harmonics have shorter wave lengths and so the growth rate is very small and in fact, there is a cut off below a certain wavelength due to FLR effects. Thus the wave energy would be dissipated from large wavelength waves to short wavelength waves as a result of which the waves with large wavelength would also become stable.

Thus the growing R-T modes evolve into coherent, almost harmonic, travelling stationary solutions. However, if the nonlinear stationary amplitude is sufficiently large, it destroys the phase-coherence (as is obvious by eq. 35 and arguments followed) which the growing modes display when the amplitude is small. This, in turn, distorts 
the model structure (sinusoidal) as energy is interchanged between fundamentals and harmonics.

\section{Discussions and conclusions}

We have seen that the R-T instability-driven modes can evolve into a coherent, nonlinear, travelling stationary waves having a constant phase velocity due to nonlinear interaction with the higher harmonics generated by the fundamental unstable R-T mode.

It is found from the linear theory that the growth rate (15) consists of two terms; one involving the vertical drift velocity $v_{D}$ due to the electric field and the other gravity. In the situation when the electric field is either significantly weak or absent, it has been suggested that spread $F$ must occur at a higher height. However, if one considers the enhanced value of the electric field that is observed, this character of spread $F$ is changed. It is found that spread $F$ will occur even at a lower height in this case. For example, if $v_{D}$ is of the order of $100 \mathrm{~m} / \mathrm{s} . v_{i} v_{D} / L$ is comparable to $g / L$. From the experimental observations, Farley et al (1970) have shown that it is the altitude threshold which is significant for the spread $F$ onset together with the sharper gradient while Rastogi and Woodman (1977) have reported that this threshold is not essential for spread $F$ if there exists a large electric field. It is very interesting that both these observations are in complete agreement with the theoretical prediction just mentioned above.

It has been shown that the leading nonlinear effects appear in the weakly nonlinear case through $V_{L}$ which essentially gives the FLR corrections. The FLR correction effects reduce the growth rate and contribute to the real part of the frequency and so the phase velocity can increase with increasing FLR correction effects. For $V_{L}<u$ $<V_{0}$, it has been shown that the coherent, almost sinusoidal, travelling stationary solution of the equations describing R-T instability driven modes are found to exist in the equatorial $F$ region. Physically, the mechanism of stabilizing these modes seem to be the nonlinear coupling between the growing fundamental wave and its higher harmonics, which gives rise to the dissipation of energy associated with the fundamental to higher harmonics.

The recent satellite observations (Calvert and Schmidt 1964; McClure 1973; Dyson et al 1974) do indeed provide the experimental evidence for such nonlinear travelling wavelike structures of equatorial spread $F$ irregularities over a large scale sizes ( $<70 \mathrm{~m}$ to $20 \mathrm{kms}$ ). Dyson et al $1(974)$ also observed that at times the power spectra of equatorial spread $F$ irregularities show that the power is contained only within a limited scale size band and that the wave form of the irregularities is composed of the fundamental and harmonics. This limited scale size range turns out to be the fundamental and the first harmonic. The scale size of the fundamental is around $7.5 \mathrm{~km}$ which seems to agree well with the typical wavelength of a R-T mode strongly excited in the equatorial $F$ region. Presumably, this is the scale size at which the dissipation of energy associated with the fundamental to the higher harmonics occur due to nonlinear interactions between the fundamental and its higher harmonics and thereby giving energy to smaller irregularities with scale sizes down to $70 \mathrm{~m}$ or even less. Since smaller wavelength modes have finite cut off in growth, they are stabilized 
at some finite amplitude. All these waves then must travel with phase velocity $u_{0}$ given by (34).

Further, the experimental evidence in favour of our results, seem to arise from rocket observations also (Kelley and Carlson 1974; Kelley et al 1976). These authors reported that the spread $F$ irregularities following the onset, developed into coherent, steepened structures in a time period of $\sim 30 \mathrm{~min}$. The result obtained here appears to show a good agreement with these observations. The typical growth time of $\sim 10^{3}$ sec for the R-T modes estimated in the $F$ region, also seems to agree well with the observed growth time of the irregularities. Also the fact that such nonlinear structures are observed only in the equatorial spread $F$ region is of considerable significance, since it ties up well with the view that the R-T instability is the main source of irregularities, whereas a different mechanism (Perkins 1973) seems to operate for the generation of spread $F$ irregularities at higher latitudes (Calvert and Schmidt 1964; McClure 1973; Dyson et al 1974).

There are also observations of irregularities at high altitude regions $(\sim 700$ to 1000 $\mathrm{kms}$ ) by topside sounders at wavelengths of the order of 100 metres to $20 \mathrm{kms}$ (Calvert and Schmidt 1964; Dyson et al 1974) and by radar backscatter at the wavelength of 3 metres (Farley et al 1970; Woodman 1973). Although the R-T instability is linearly stable on the topside, these irregularities may be explained by upwelling of the low density regions which may populate the topside (Kelley et al 1976). A more detailed work in this direction will be carried out in near future.

\section{References}

Balsley B B, Haerendel G and Greenwald R A 1972 J. Geophys. Res. 775625

Bogoliubov N N and Mitropolsky Y A 1961 Asymptotic methods in theory of nonlinear oscillations (Delhi: Hindustan Pub. Corp.) p. 51.

Booker H G and Wells H W 1938 Terr. Magn. Atmos. Elect. 43249

Calvert W and Schmidt C W 1964 J. Geophys. Res. 691839

Chaturvedi P K and Kaw P K 1975 Geophys. Res. Lett. 2381

Cunnold D M 1969 J. Geophys. Res. 745709

Dungey J W 1956 J. Atmos. Terr. Phys. 9304

Dyson M L, McClure J P and Hanson W B 1974 J. Geophys. Res. 791497

Farley D T 1974 Rev. Geophys. 12285

Farley D T, Balsley B B, Woodman R F and McClure J P 1970 J. Geophys. Res. 757199

Hanson W B and Sanatani S 1973 J. Geophys. Res. 781167

Herman J R 1966 Rev. Geophys. 4255

Holtet J A, Maynard N C and Heppner J P 1975 EOS Trans AGU (Abst.) 56418

Hudson M K and Kennel C F 1974a UCLA-Rept. PPG-179

Hudson M K and Kennel C F 1974b UCLA-Rept. PPG-201

Hudson M K and Kennel C F 1975a J. Plasma Phys. 14121

Hudson M K and Kennel C F 1975b J. Plasma Phys. 14135

Hudson M K and Kennel C F 1975c J. Geophys. Res. 804581

Hudson M K, Kennel C F and Kaw P K 1973 EOS Trans. AGU (Abstract) 541147

Johnson M H and Hulbert E O 1950 Phys. Rev. 79802

Kelley M C and Carlson C W 1974 EOS Trans. AGU 55381

Kelley M C et al 1976 Geophys. Res. Lett. 3448

McClure J P 1973 Paper presented at first Lloyd Berkner Symp. AGU Univ. Texas Dallas Texas

Perkins F W 1973 J. Geophys. Res. 78218

Rastogi R G and Woodman R F 1977 Communicated to Ann. de. Geophys.

Reid G C 1968 J. Geophys. Res. 731627

Rishbeth H and Garriott O K 1969 Introduction to ionospheric physics (New York : Academic Press)

Rosenbluth M N, Krall N and Rostoker N 1962 Nucl. Fusion Suppl. 1143

Woodman R F 1973 A review paper presented at first Lloyd Berkner symp. AGU Univ. Texas Dallas Texas 